\title{
In people and pets in Grenada - do owners practice alternative and traditional medicine on both people and pets?
}

\author{
Monique Fitzpatrick*, Brittany Perfetto, Jose Hernandez, Satesh Bidiasee \\ School of Veterinary Medicine, St. George's University, Grenada
}

Received: April 10, 2018

Accepted: May 18, 2018

Online Published: June 11, 2018

DOI: $10.5430 /$ jbei.v4n $1 \mathrm{p} 47$

URL: https://doi.org/10.5430/jbei.v4n1p47

\begin{abstract}
Introduction: Alternative and traditional medicine has been used in various cultures across the world for hundreds of years. Many regions of the world, including the Caribbean Islands, use traditional medicine practices such as charcoal for poisoning, seeds for deworming, salt for lesions, spices and herbs for inflammation, and engine oil for mange. In communities where biomedical resources are limited, alternative medicine is more cost effective and attainable for both people and their pets. Although conventional medical practices have a scientific basis, its options are often limited and such practices and treatments have physiological side effects that must be considered.

Objective: The objective of this study is to identify whether people in Grenada practice traditional medicine, and if these practitioners are also implementing similar practices on their companion animals and themselves. It is hypothesized that many citizens of Grenada prefer the use of traditional medicine over conventional medicine for both themselves and their pets. Conventional medicine is a system where medical professionals, such as doctors, nurses and pharmacists, treat symptoms and diseases with the use of drugs, radiation or surgery. Traditional medicine is the health practices, approaches, knowledge and believes by many cultures in which they incorporate plant, animal and mineral based medicines to treat, diagnose and prevent illnesses.

Methods: In order to test this hypothesis, we conducted a cross sectional study using a questionnaire to collect data from pet owners and determine whether they practice traditional medicine - and if so, identify which specific practices were used and how they were being implemented (strictly on themselves, only on their pets, or both).

Results: We found that many people use alternative medicine practices on themselves and both themselves and their pets, but few use them on just their pets.

Conclusions: This study can offer us a more comprehensive understanding of alternative medicine, thereby allow us to engage pet owners in this area of healthcare. Veterinarians can be better informed of the alternative medical practices that their clients may be utilizing, which will allow them to educate the owners by offering their guidance and expertise in approaching traditional medical practices - and perhaps offer alternative medical solutions when appropriate. Furthermore, it will allow veterinarians to formulate more comprehensive patient histories in veterinary clinics around Grenada.
\end{abstract}

Key Words: Traditional medicine, Caribbean, Alternative medicine, Grenada, Survey

*Correspondence: Monique Fitzpatrick; Email: mfitzpat@ @gu.edu; Address: School of Veterinary Medicine, St. George’s University, Grenada. 


\section{INTRODUCTION}

Alternative and traditional medicine is widely used in many cultures across the world for hundreds of years. ${ }^{[1]}$ Traditional medicine refers to the health practices, approaches, knowledge and beliefs of cultures that incorporate plant, animal and mineral based medicines, spiritual therapies, manual techniques and exercises which are used singularly or combined to treat, diagnose and prevent illnesses. ${ }^{[14]}$ Many regions of the world, including the Caribbean Islands, use traditional medicine practices such as charcoal for various poisons and toxins, plant seeds for deworming, salt, oil, and mud water for skin lesions, spices and herbs for inflammation, and engine oil for the treatment of mange.

Charcoal is typically administered to animals whom have been poisoned by any number of toxic ailments including grapes, chocolate, caffeine, alcohol, garlic/onions, medications, pesticides and insecticides-among others. ${ }^{[2]}$ Charcoal naturally binds to and absorbs toxins in the gastrointestinal and thus detoxifies the body. For maximal absorption, activated charcoal should be given as soon as possible after exposure, as delayed administration can reduce effectiveness. ${ }^{[3]}$ Although this is considered a traditional medicine practice, charcoal is used in many veterinary clinics as an emetic. ${ }^{[2]}$

Plant seeds are commonly used for deworming animals. These therapeutic seeds can be found among plants native to tropical climates-papaya seed, neem tree tea seed, and mammy seed. ${ }^{[4,5]}$ They act as a preventative, as well as treatment for the expulsion of intestinal worms by inhibiting parasitic enzymes. ${ }^{[4]}$ These seeds are easily attainable and thus provide a cost effective treatment for intestinal parasites in countries such as Grenada.

Skin lesions followed by secondary infections are quite common among animals in Grenada, and in climates alike. There are various remedies available for treatment of disease of the skin. Common remedies include sulfur spring water, mud water, salt water with oil as well as laundry detergent-all of which are applied topically to the affected areas. Such treatments have evidence for clearing skin lesions and infections due to their antimicrobial and anti-inflammatory properties. ${ }^{[6-8]}$

Spices and herbs for have long been known to effectively reduce inflammatory processes in the body. Nutmeg oil alleviates chronic inflammatory pain through the inhibition of COX-2 expression and substance $\mathrm{P}$-molecules that normally induce inflammation. ${ }^{[9]}$ Clove is used for boils, sores, local edematous swellings, inflammation and pain and possesses anti-inflammatory, anti-nociceptive and anti-pyretic properties. ${ }^{[10]}$ Moreover, extensive research has indicated that the leaves and stems of Crescentia cujeta have antiinflammatory and antibacterial properties - thus, the plant extract has therapeutic potential against bacterial infection and disease processes of the body. ${ }^{[11]}$

One remedy that is commonly used on both people and animals is engine oil for the treatment on sarcoptic and demodectic mange. This treatment has shown to be effective in clogging the pores of the epidermis while suffocating the parasitic mites within the hair follicles. ${ }^{[12]}$ Engine oil is a common item to most people and so the use of engine oil for mange is expected to be widely used.

In communities where biomedical resources are limited, alternative medicine is more cost effective and attainable for both people and their pets. ${ }^{[13]}$ Although conventional medical practices have a scientific basis, its options are often limited and such practices and treatments have physiological side effects that must be considered. ${ }^{[1]}$ In countries such as Grenada, pet owners may opt to use alternative medicine practices instead of using veterinary care but the effects of many of these practices are no known.

We hypothesize that if citizens of Grenada are aware of traditional medicine practices, then they will prefer the use of those traditional medicine practices over conventional medicine for both themselves and their pets.

\section{Methods}

The study population for this project was adults 18 or older who reside in Grenada. The inclusion criteria for this study are people who have resided in Grenada with their small animal pet(s) for more than 6 months. The sample size for this study was 30 . The participants were recruited from the main bus terminal in St. George's, a free veterinary clinic in St. Andrews, the St. George's University Small Animal clinic, and some local restaurants. Sampling took place from March 2017-October 2017. The principle investigators walked around the study sites and approached people, introducing themselves and then asking whether the person would like to participate in the study. All people who agreed to participate were be given the survey on whether they used alternative medicine practices on themselves, their pets, or both. The survey contained closed and open-ended questions so that if any alternative medicine approaches are not listed, participants could choose to include those practices in their response. The survey took about 15-20 minutes to complete and was be completed on an electronic device. The surveys did not contain personal identifiers and the responses were be stored on the electronic device for analysis. 


\section{RESULTS}

The results from this study are limited because of the small number of participants. Demographic questions show a diverse population was sampled with an almost even split between the 2 broad age categories and gender, as shown in Table 1.

Table 1. Demographics

\begin{tabular}{llllll}
\hline \multirow{2}{*}{ Groups } & \multicolumn{2}{l}{ Age Range } & & \multicolumn{2}{l}{ Gender } \\
\cline { 2 - 3 } & $\mathbf{1 8 - 4 0}$ & $\mathbf{4 1 - 7 0}$ & & Male & Female \\
\hline Survey Results & 17 & 13 & & 17 & 12 \\
\hline
\end{tabular}

There were specific questions asked in the survey which identified a natural remedy and then what it was used for. As an example shown in Figure 1, 16 out of 30 (53.3\%) responses said they used saltwater and oil for skin lesions on themselves and their pets and only 1 out of 30 (3.3\%) responses said that it was used on their pet alone. The following questions then asked whether they had seen other natural remedies, like detergent for similar skin lesions (on pets = $13.3 \%$, on both $=16.7 \%$, no $=70 \%$ ), sulfur mud (on humans $=33.3 \%$, on pets $=6.7 \%$, on both $=16.7 \%$, no $=43.3 \%$ ). Soft candle was identified as a natural remedy available in Grenada. Questions on soft candle included if it was used for pain (on humans $=63.3 \%$, on both $=3.3 \%$, no $=33.3 \%$ ), for swelling (on humans $=40 \%$, on both $=3.3 \%$, no $=33.3 \%$ ), for wounds (on humans $=40 \%$, on both $=3.3 \%$, no $=56.7 \%$ ), and for infections (on humans $=20 \%$, on both $=3.3 \%$, no $=$ $76.7 \%)$. Then there were questions on nutmeg used for pain (on humans $=63.3 \%$, on both $=13.3 \%$, no $=23.3 \%$ ), and for swelling (on humans $=56.7 \%$, on both $=13.3 \%$, no $=$ $30 \%)$. The participants were asked about two treatments for mange, motor oil (on pets $=50 \%$, no $=50 \%$ ) and calabash (on humans $=6.7 \%$, on pets $=10 \%$, on both $=3.3 \%$, no $=80 \%$ ). For the treatment of poison, the participants were asked if they used charcoal (on pets $=13.3 \%$, on both $=20 \%$, no $=66.7 \%$ ). Some alternative medicine practices were used on humans often, like shown in Figure 2 with the use of clove for pain. 19 out of $30(63.3 \%)$ responses indicated that clove was only used for pain in humans and 7 out of 30 (23.3\%) did not use clove for pain on either pets or themselves with 4 out of $30(13.3 \%)$ using it on both. Other questions asked on clove was if it was used for swelling (on humans $=23.3 \%$, on both $=3.3 \%$, no $=73.3 \%$ ) and for boils (on humans $=6.7 \%$, no $=93.3 \%)$. For the treatment of worms, we asked about the use of lard (on humans $=3.3 \%$, on pets $=10 \%$, on both $=6.7 \%$, no $=80 \%$ ), papaya seeds (on humans $=13.3 \%$, on both $=16.7 \%$, no $=70 \%$ ), neem tree tea (on humans $=3.3 \%$, on pets $=3.3 \%$, no $=93.3 \%)$, and mammy seeds $($ no $=100 \%)$. The last two close-ended questions asked if any plants had been used for diarrhea (on humans $=20 \%$, on pets $=3.3 \%$, on both $=6.7 \%$, no $=70 \%$ ) or for fever (on humans $=26.7 \%$, on pets $=3.3 \%$, no $=66.7 \%$, no answer $=3.3 \%$ ).

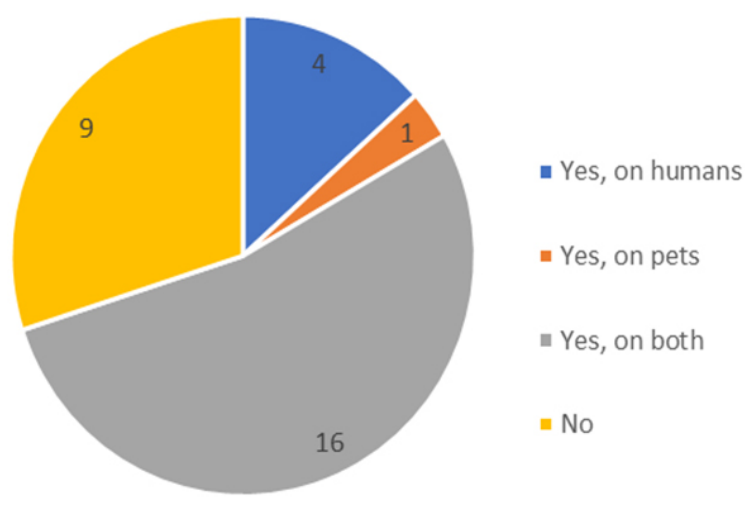

Figure 1. Saltwater and oil for skin lesions

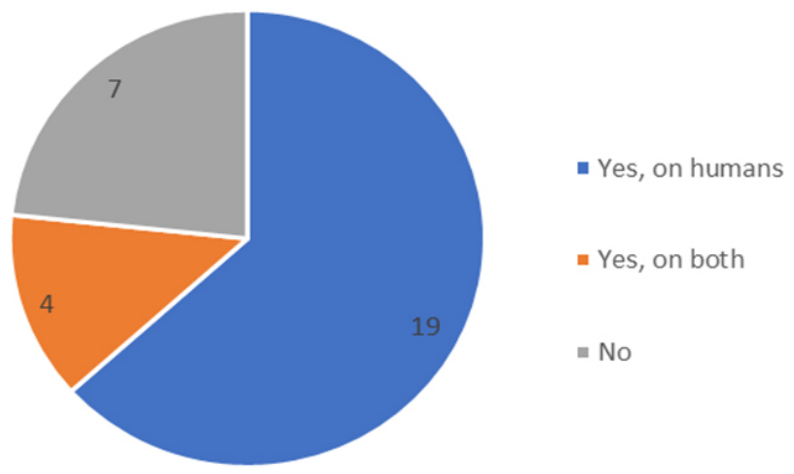

Figure 2. Clove for pain

Open-ended questions were asked about each natural remedy and then at the end to give each participant the opportunity to identify any alternative medicine practices that they had used but were not asked about. Plants that were used for diarrhea were lemon juice ( 2 responses) and powdered bark of a red elm tree (3 responses). Plants that were used for fever were hay tea with parsley (1 response) and yarrow (6 responses).

As shown in Table 1, demographic questions were asked of the survey participants. The broad age ranges were 18-40 and $41-70$ but in the survey they were further narrowed into 18-30, 31-40, 41-50, 51-60, and 61-70. Gender was also asked and the participants could also choose not to answer.

As shown in Figure 1, most participants used saltwater and oil for skin lesions on themselves and their pets $(16 / 30=53.3 \%)$. Few only used saltwater and oil on either themselves $(4 / 30=$ $13.3 \%)$ or their pets $(1 / 30=3.3 \%)$ and then some participants did not use this alternative medicine practice at all $(9 / 30=$ $30 \%)$.

As shown in Figure 2, most participants only used clove for pain on themselves $(19 / 30=63.3 \%)$. Many participants did 
not use clove for pain at all $(7 / 30=23.3 \%)$ and few used clove for pain on both themselves and their pets (13.3\%). No participants used clove for pain only on their pets.

\section{Discussion}

In various areas of world, including the Caribbean, many people use traditional and alternative medicine to take care of themselves and their animal companions. Our findings suggest that the citizens of Grenada choose alternative medicine for themselves and their animal companions instead of traditional medicine. Several significant practices that are seen and noted in the survey include using salt water and oil for skin lesions, clove for pain, charcoal for toxin ingestion, nutmeg for inflammation and sulfur for skin issues.

In this study, we asked the citizens of Grenada about various practices that are seen on this island. These questions were asked in the form of a survey. Other demographics, other than what is included in Table 1, were also asked including highest level of education (primary $=16.7 \%$, secondary $=43.3 \%$, undergraduate $=33.3 \%$, professional $/$ graduate $=$ $3.3 \%$, none $=3.3 \%)$, number of persons in household (1 persons $=10 \%, 2$ persons $=26.7 \%, 3$ persons $=16.7 \%, 4$ persons $=16.7 \%, 5+$ persons $=26.7 \%$, no answer $=3.3 \%$ ), employment status (employed $=76.7 \%$, not employed $=$ $26.7 \%$, no answer $=3.3 \%$ ), and if they were part-time or full-time (full-time $=66.7 \%$, no answer $=33.3 \%$ ). Some questions were also asked regarding pets and veterinary care which included if they had any experience working with animals (yes $=70 \%$, no $=26.7 \%$, no answer $=3.3 \%$ ), if they currently owned a pet (yes $=93.3 \%$, no $=3.3 \%$, no answer $=$ $3.3 \%$ ), if their pets were indoor or outdoor (strictly indoor $=6.7 \%$, strictly outdoor $=66.7 \%$, both $=23.3 \%$, no answer $=3.3 \%$ ), if they had ever received veterinary care (yes = $46.7 \%$, no $=53.3 \%$ ), how regularly they received veterinary care (yes $=50 \%$, no $=50 \%$ ), if they sought veterinary care when the pet was ill (yes $=63.3 \%$, no $=33.3 \%$, no answer $=$ $3.3 \%$ ), and if they ever used natural remedies on their pets (yes $=56.7 \%$, no $=43.3 \%$ ). Each participant was also asked if they used natural remedies on themselves (yes $=53.3 \%$, no $=26.7 \%$, sometimes $=16.7 \%$, no answer $=3.3 \%$ ). One practice that particularly stands out is the use of salt water and oil for skin lesions. Majority of the participants (53.3\%) used salt water and oil on both themselves and their animal companions, which is seen in Figure 1. On the contrary, when it came to the use of clove for pain (see Figure 2), most of the participants only used it on themselves (63.3\%), few used it on both themselves and their pets (13.3\%) and several did not use clove at all (23.3\%). The open-ended questions helped to identify potential additions to another survey to further gain results from a more representative population.
We also observed that many of the pet owners did not regularly seek or provide veterinary medical assistance for their pets unless it was either an emergency or a very serious medical condition, which was asked in the survey. This may, in part, be due to the economic pressures and limited income most Grenadians face, but it is evident that it also has a cultural aspect to it as well. The lack of proper guidance and knowledge and the fact that Grenadians rely heavily on "general knowledge" passed down from generation to generation when it comes to the physiology, husbandry, and welfare of pet animals (i.e. dogs and cats) is the other great contributing factor.

The greatest problem with this study, however, was the small sample size that we obtained. Due to the small number of participants, the results do not fully represent the populous of Grenada and their practices. Many citizens did not want to participate in the interview which resulted in the small sample size in this study. However, we were still able to get some insight on the medical practices that are done amongst the populous. Another problem that we encountered during this study was that many citizens indicated that they did not know the names of the items they used while we were performing the surveys. However, they were able provide a description of the items. So, we were able to take note on the descriptions and determine if they match up with the items described in the survey. In a future study, it is recommended to obtain a larger sample size in order to properly represent the populous of Grenada. A larger sample size could be accomplished by traveling to areas in Grenada where there is a heavier population such as the town of St. George's, particularly the bus stop located in this town. Many citizens use public transportation to get around the island, so this would be a prime location to interview more citizens. It is also recommended to provide photos of the myriad items described in the survey so that it is easier to identify the items that are used in medical practices amongst the populous.

Many of the formulations and reagents used in alternative medicine are not subjected to high levels of scrutiny or quality control, especially in countries such as Grenada where the resources are either unavailable or are too expensive to be cost efficient to the producer. This is a matter of ample concern, one which can negatively impact or even jeopardize the health and welfare of both humans and their pets exposed to these products due to the risk of contamination with harmful pathogens and/or chemicals during the formulation and preparation of such products. Another factor which increases the risks of these products is the unknown concentrations, potency, or toxicity of both active and inactive compounds contained in them. In addition, the actual health benefits and efficacies of the vast majority of these formulations and 
practices are largely debated, but some do show promise and others have been found to possess various beneficial pharmacological effects. Therefore, it is of utter importance to seek consult from a medical professional that possess an extensive knowledge and understanding of human and/or animal physiology, pathology, pharmacology, and of the materials and methods used in the numerous branches of this field of medicine before engaging in or receiving such services, especially if the individual has an underlying or currently manifested illness or condition. In conclusion, we saw that the locals that are using still alternative medical practices mainly use it on themselves and not their pets. This could because many of the local alternative medical practices include home remedies such as clove for pain, sulfur for skin conditions and others. Many of these conditions are either hard to observe in our companion animals or present differently than humans do, which greatly influences why we did not such almost any crossover of medical practices between humans and their pets. Another reason is that the newer generations of Grenadians may prefer the use of pharmaceuticals for the treatment of their minor ailments instead of natural remedies and alternatives. Again, this may also be due to the fact that many citizens do not seek out medical care unless their pets are very ill. It is also important to note that those many citizens have food animals such as sheep, goats and chickens as pets as well as a food source, so the way these animals are treated differs greatly from the way a dog or a cat would. High costs and scarcity of products such as pharmaceuticals, basic and specialized healthcare services, and trained or experienced personnel are the main contributing factors to why many individuals, locally and globally, prefer or are forced to seek for alternative medical practices, due to the fact they are often cheaper and more accessible. This observation lets us appreciate and understand one of the many reasons why such practices are still wildly used today in many countries around the globe.The line of study is important to providing insight to veterinarians and general practitioners about alternative practices that are seen. This will allow them to educate their clients about these practices when they deem them important for the care of their companion animals.

\section{Conclusion}

The aim of this survey is to conduct and analyze compiled data, in hopes to uncover the extent of the alternative medical practices currently being used by the people of Grenada, on themselves and their animal companions. With the data we were able to identify various techniques, plants/extracts/compounds, and procedures pertaining to the expansive field of alternative medicine practiced by the local populous. This data also provided us a means to engage pet owners in this area of healthcare. Furthermore, it also aids in raising awareness and informing local veterinarians and general medical practitioners of the alternative medical practices that their clients might be employing on themselves and their pets.

With this study, we hope to open a window of opportunity and progress, by allowing veterinarians and general medical practitioners to better educate their clients about the potential benefits, risks, adverse effects, and contraindications some of these practices might possess, induce, or exacerbate. Furthermore, it may assist veterinarians to supplement or offer alternative medical solutions when appropriate. The information gathered will also aid researchers conducting or planning future studies of this nature and help determine which alternative practices are beneficial and which are not. Finally, it will have a positive impact and reinforcement on doctor-patient communications by assisting veterinarians and general medical practitioners in acquiring a more comprehensive patient history (and possibly diagnosis and/or treatment as well) in clinics and hospitals worldwide.

\section{ACKNOWLEDGEMENTS}

We would like to thank Jessica Austin for helping us with the initial phases of the project design and background research. We would also like to thank Dr. Heidi Janicke, Dr. Melania Crisan, and Dr. Richard Kabuusu for giving feedback and guidance while completing this research.

\section{REFERENCES}

[1] Kalsi DS, Ryan TJ. The integration of complementary and alternative medicine: the way forward for the health of skin and gut. Current Science. 2016; 111: 343-350. https ://doi.org/10.18520/cs/ v111/i2/343-350

[2] Bates N, Rawson-Harris P, Edwards N. Common questions in veterinary toxicology. Journal of Small Animal Practice. 2015; 56: 298306. PMid:25728477. https ://doi .org/10.1111/jsap. 12343

[3] Eddleson M, Juszczak E, Buckley NA, et al. Multiple-dose activated charcoal in acute self-poisoning: a randomized controlled trial. The Lancet. 2008; 371: 16-22. https ://doi .org/10.1016/S0140-6 736 (08) 60270-6

[4] Mitchell MJ, Smith SL, Johnson S, et al. Effects of the neem tree compounds azadirachtin, salannin, nimbin, and 6desacetylnimbin on ecdysone 20-monooxygenase activity. Archives of Insect Biochemistry and Physiology. 1997; 35: 199209. https://doi.org/10.1002/(SICI) 1520-6327(1997) 3 $5: 1 / 2<199::$ AID-ARCH18>3.0.CO;2-6 
[5] Hammond JA, Fielding D, Bishop SC. Prospects for plant anthelmintics in tropical veterinary medicine. Veterinary Research Communications. 1997; 21: 213-228. https://doi.org/10.1023/A : 1005884429253

[6] Barrager E, Veltmann JR, Schauss AG, et al. A multi-centered, openlabel trial on the safety and efficacy of methylsulfonylmethane in the treatment of seasonal allergic rhinitis. Journal of Alternative and Complementary Medicine. 2002; 8: 167-73. PMid:12006124. https://doi.org/10.1089/107555302317371451

[7] Gomes CS. Healing and edible clays: a review of basic concepts, benefits and risks. Environmental Geochemistry and Health. 2017; 1 27. PMid:26817925. https://doi.org/10.1007/s10653-016 $-9903-4$

[8] Gambichler T, Terras S, Skrygan M. Cyclooxygenase 2 expression and apoptosis in normal and psoriatic epidermis models exposed to salt water soaks and narrowband ultraviolet B radiation. Journal of the European Academy of Dermatology and Venerology. 2015; 29: 180183. PMid:24341417. https://doi.org/10.1111/jdv.12345
[9] Zhang WK, Tao S, Li T, et al. Nutmeg oil alleviates chronic inflammatory pain through inhibition of COX-2 expression and substance P release in vivo. Food \& Nutrition Research. 2016; 60. https://doi.org/10.3402/fnr.v60.30849

[10] Taher Y, Samud AM, El-Taher FE, et al. Experimental evaluation of anti-inflammatory, antinociceptive and antipyretic activities of clove oil in mice. Libyan Journal of Medicine. 2015; 10. https://doi.org/10.3402/ljm.v10.28685

[11] Parvin MS, Das N, Jahan N, et al. Evaluation of in vitro antiinflammatory and antibacterial potential of Crescentia cujete leaves and stem bark. BMC Research Notes. 2015; 8: 412. https ://doi. org/10.1186/s13104-015-1384-5

[12] Gakuya F, Ombui J, Heukelbach J, et al. Knowledge of Mange among Masai Pastoralists in Kenya. PLoS One. 2012; 7. https: //doi.org/10.1371/journal. pone.0043342

[13] Aarons DE. Medicine and its alternatives. Hastings Center Report. 1999; 29: 23-27. https ://doi .org/10.2307/3528063

[14] “Traditional Medicine." Health Topics A to Z. Available from: www . allcountries.org/health/traditional_medicine.html 